\title{
Case of true cyclopia
}

\author{
HANNA J GARZOZI AND SUSAN BARKAY \\ From the Department of Ophthalmology, Central Emek Hospital, Afula, Israel
}

SUMmARY True cyclopia is a rare anomaly in which the organogenetic development of the two separate eyes is suppressed. A case of true cyclopia with normal karyotype is presented. There was a history of the use of an intrauterine device for contraception and of drug ingestion during early pregnancy. An anatomicopathological study of the monster with detailed presentation of the cyclopean eye is reported. The possible causes of cyclopia with particular relation to this case are discussed.

Cyclopia is an unusual anomaly in which the anterior brain and the midline mesodermal structures develop anomalously. The orbital region is grossly deformed, resulting in the formation of a central cavity 'pseudoorbit,' with absence of nasal cavity and presence of a rudimentary proboscis above the pseudo-orbit. If two globes are found in a different degree of fusion in the pseudo-orbit, the condition is called synophthalmos. A much rarer anomaly is true cyclopia, wherein only one eye is present. ${ }^{12}$

Exposure to drugs or to other potentially teratogenic environmental factors during organogenesis has been regarded as the basis of this anomaly. These factors were ionic radiation, contraceptives, viraemia plus corticosteroids and salicylates, rubella vaccine, antibiotics, and amidopyrine (aminopyrine).$^{34}$

Genetic errors with chromosomal abnormalities such as trisomy-D, monosomy-G mosaicism, translocation affecting chromosome 3 and group $C$ chromosome, and chromosome 10 short arm deletion have also been recorded in cyclopia. ${ }^{15-13}$

In most of the recent reports there was no detailed description of the histopathological findings of the eyeball with reference to the presence of true cyclopia or synophthalmos. ${ }^{3-13}$ We give here a detailed presentation of a case of true cyclopia where complete fusion of the two eyes was found, together with a detailed description of the histopathological findings in the pseudo-orbit, with particular reference to the history of the pregnancy, drug ingestion, and possible environmental factors during pregnancy.

\section{Case report}

The cyclop was a female infant weighing $2820 \mathrm{~g}$ born

Correspondence to Hanna J Garzozi, MD, Department of Ophthalmology, Central Emek Hospital, Afula 18101, Israel. on the 13 August 1982 at 33 weeks gestation with vertex presentation; she lived for about 30 minutes (Fig. 1). The birth was the product of the ninth pregnancy of a 34-year-old Arab woman, para 9, married to her first cousin on her father's side. All her previous pregnancies and deliveries were normal. Her sixth delivery was twins.

About four years before her last pregnancy she was injected twice, at six-month intervals, with medroxyprogesterone acetate for birth control, after which she had her eighth pregnancy and delivery without any complications. Her last menstrual period before the last pregnancy, was on 23 December 1981. On 31 December 1981 an intrauterine device made of polyethylene and copper was inserted, and a course of tranexamic acid (Hexacaprone) one tablet three times daily for five days and ampicillin $500 \mathrm{mg}$ four times daily were given for two weeks to prevent haemorrhage and infection after introduction of the intrauterine device. The pregnancy progressed with polyhydramnios, and three weeks before birth the mother was exposed to $x$ rays for the diagnosis of swelling and tenderness in her right calf.

On 13 August 1982 a female cyclopean monster was born spontaneously with cephalic (vertex) presentation. She moved her hands and legs moderately and had a corneal reflex, as was reported by the obstetrician. She died about 30 minutes after birth from respiratory distress. The placenta was expelled 20 minutes later and weighed $650 \mathrm{~g}$. There were three blood vessels in the cord.

Chromosomal studies of blood cell cultures from the cord and the peripheral blood taken from the parents showed a normal karyotype.

NECROPSY

On post-mortem examination malformations were 




Fig. 1 Newborn cyclopian monster.

discovered mainly in the face and the central nervous system. In the other systems the only defect was a patent ductus arteriosus. The outer appearance of the body and limbs was normal; anatomicopathological study of the other organ systems, including respiratory, digestive, urogenital, and endocrine, showed normal organs.

The head was well shaped. In the face (Fig. 1) there was a single median diamond shaped orbit with a single well developed globe covered by two pairs of eyelids which were developed laterally and joined into the midline by a notch or indentation. There were four lacrimal puncta. A proboscis measuring 25 $\mathrm{mm}$ in length and $10 \mathrm{~mm}$ in diameter with a single blind end orifice was attached to the superior aspect of the orbit. There were no abnormalities of the palate, pharynx, and larynx. The nose was absent.

Examination of the central nervous system revealed an $80 \mathrm{~g}$ brain (third of the normal weight for its age) covered by a large dorsal cyst (Fig. 2). The cerebrum was not divided into hemispheres and the sulci and gyri were not as prominent or as clearly marked as in the case of a normal brain at term. There was no olfactory apparatus, no optic chiasma, and no optic tracts or pituitary gland. Only one median connec-

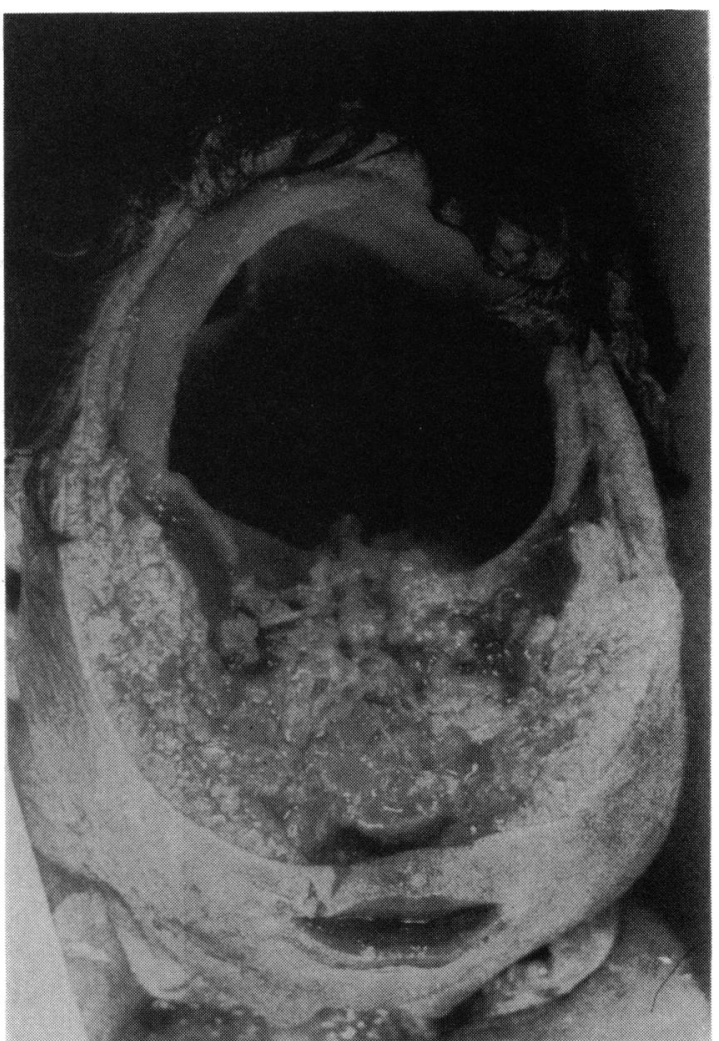

Fig. 2 Large dorsal cyst of brain.

tion was seen between a single optic foramen and the eyeball, which was presumed to be the sheaths of the optic nerve. On coronal section of the brain no midline dividing structures such as corpus callosum and septum pellucidum were found, and the common ventricle of the telencephalon was not divided into distinct lateral ventricles, but formed a large horseshoe shaped space which opened posteriorly into the large dorsal cyst.

The pseudo-orbit presented the appearance of fusion of the temporal halves of two orbits. Radiographic findings showed hypoplasia of frontal, ethmoidal, sphenoid, maxillary, and zygomatic bones and absence of nasal, vomerine, and lacrimal bones. A single optic nerve canal was present in the apex of the pseudo-orbit in the midline portion of the sphenoidal bone.

The orbit contained one well developed cyclopian eye (Fig. 3), striated muscles, hypertrophic nerves with increased number of Schwann cells, fat tissue, and connective tissue. On serial sections of the connection supposed to be optic nerve no nerve fibres were found.

Histopathological examination of the eyeball revealed a sclera of normal thickness (Fig. 4), a 


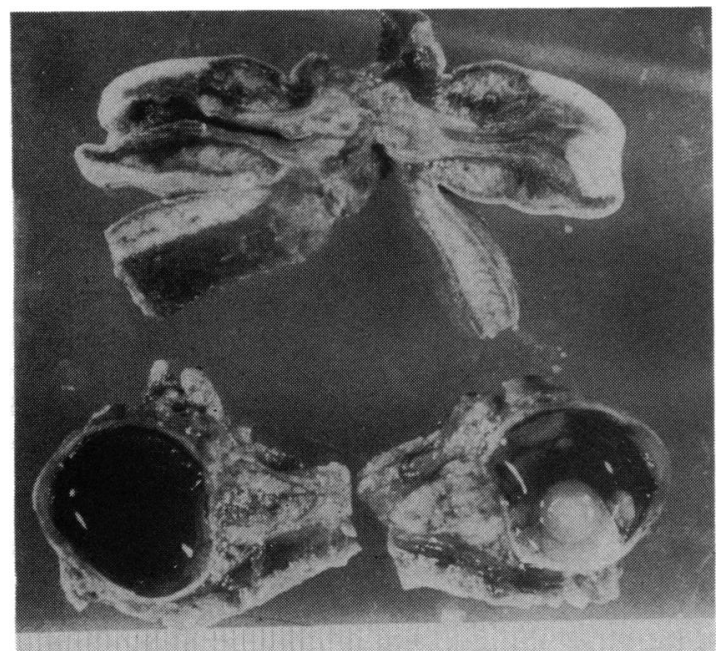

Fig. 3 Macroscopic section of the proboscis (above) and of the cyclopic eyeball (below).

hypercellular cornea, congested iris with sphincter muscle, but no dilator muscle or endothelial cells on its anterior surface were found (Fig. 5). The ciliary body presented anterior insertion of the ciliary processes, which were also short (Figs. 4, 5). The pars plana was very short, and the transformation of the ciliary pigment epithelium into retinal pigment epithelium was sudden in some places (Fig. 5). The choroid was congested, and there were some areas where it could not be recognised. The retina was disorganised and incompletely developed with areas



Fig. 4 Section of the eyeball showing anterior insertion of short ciliary processes (long arrow), and dysplastic retina in the posterior pole (open arrow). (Haematoxylin and eosin).

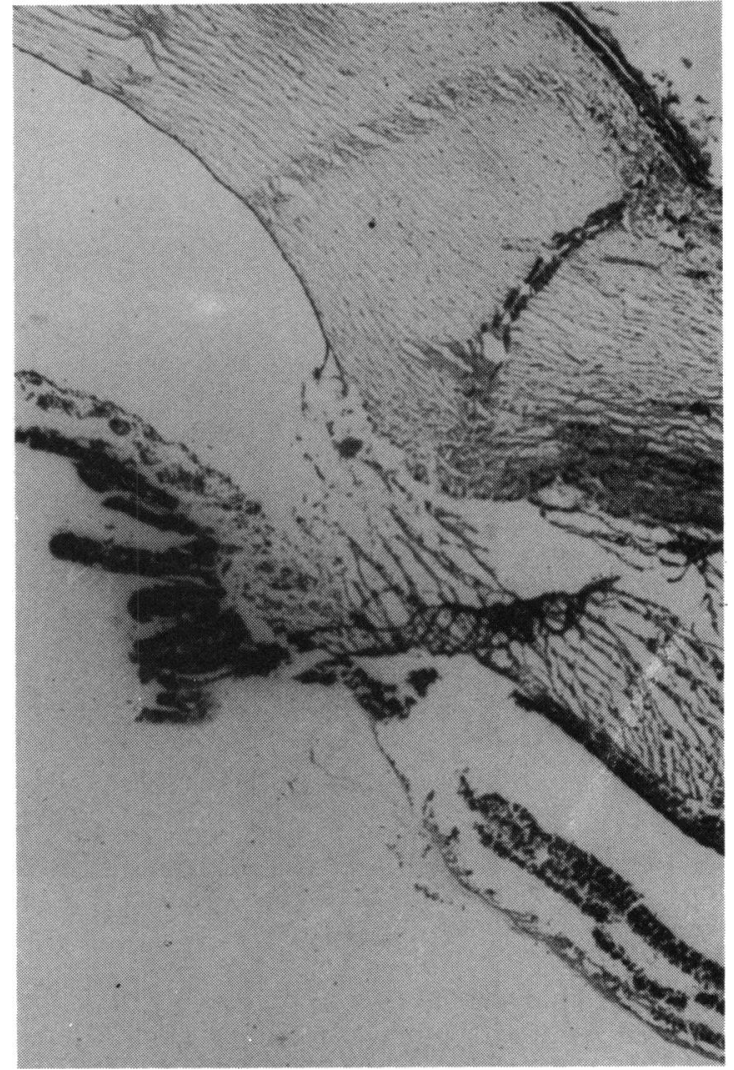

Fig. 5 Chamber angle covered by mesodermal tissue.

Anterior insertion of ciliary processes. Congested iris. Very short pars plana of the ciliary body with sudden

transformation into retina. (Haematoxylin and eosin, $\times 50$ ).

of retinal dysplasia and formation of rosettes. Only here and there were glial cells found. There were no ganglion cells or nerve fibres. In the periphery ciliary epithelium changed abruptly into retina and then changed into a primitive neuroectoderm resembling non-pigmented epithelium. Retinal tissue projected into the vitreous (Fig. 6) and in some areas into the sclera.

The angle of the anterior chamber was not open at all and Schlemm's canal could not be recognised. Mesodermal tissue covered the area to be trabeculum (Fig. 5). Histological study of the lens showed nothing abnormal.

\section{Discussion}

Cyclopia, which is an anomaly of organogenesis of the eye, results from arrest of development of the anterior end of the neural plate. ${ }^{14} 15$ Thus cyclopia is always associated with abnormalities of the brain. The anomaly was classified by De Myer et al. ${ }^{16}$ as the 


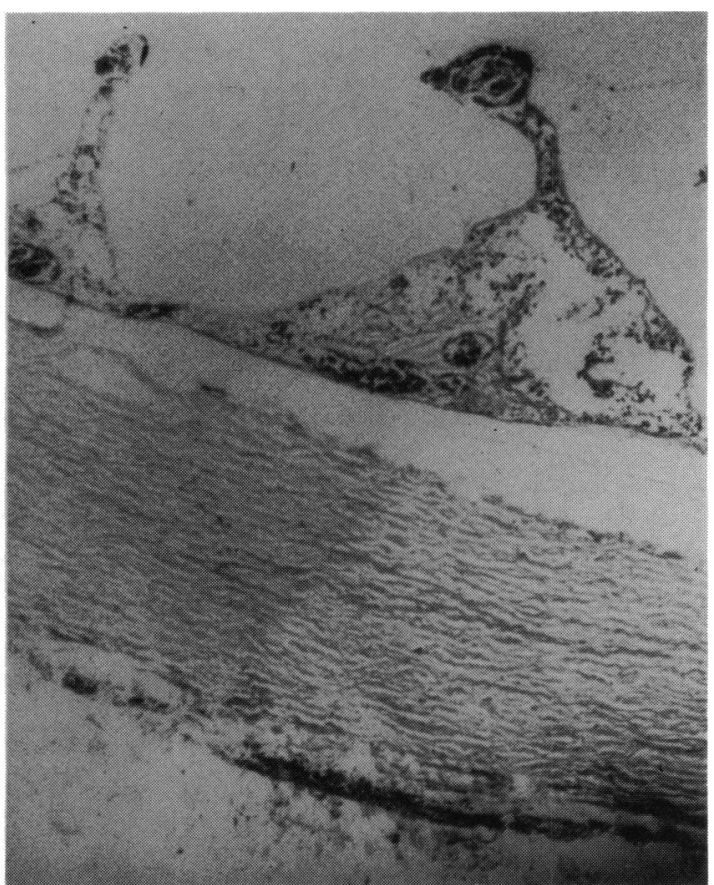

Fig. 6 Retinal projection into vitreous. (Haematoxylin and eosin, $\times 50$ ).

most extreme form of holoprosencephaly when they graded the disorders in which there is impaired midline cleavage of the embryonic forebrain. The same authors correlated the appearance of the face and the type of brain malformation in holoprosencephaly. Confirming that view, Torczynski et al. ${ }^{1}$ reported data based on the analysis of a series of 35 cases of synophthalmia-cyclopia.

In the early development of the embryo certain groups of cells (anlage) differentiate into masses destined to give rise to definite organs or tissues. Injury or abnormality of these specialised cell masses at certain critical stages during their development can result in congenital malformation of the organs or tissues. ${ }^{14}$

The two chief mechanisms are defective inheritance and an unfavourable environment. These mechanisms must act on the embryo at or before the determination of the eye anlage, and continuously thereafter by altering the interaction between the anlage and the surrounding endomesoderm. ${ }^{14}$ This action must take place before the first manifestation of the bilateral nature. In the human embryo this is at $1.8 \mathrm{~mm}$ stage or during the third week of gestation.

Our case was a female cyclopian monster born to first cousin parents. Seventeen of the 35 cases reported by Torczynski et al. ${ }^{1}$ were females, nine were males, and in the remaining nine the sex was not stated. The literature supports the predominance of cyclopean female monsters. Cohen and Gorlin" reported a case and considered the genetic aspects of holoprosencephaly. In addition to their case with parenteral consanguinity they mentioned another two cases reported by Klopfstock in 1921 and Grebe in 1954. To our knowledge our case is the fourth to be reported.

Francois ${ }^{17}$ suggested that cyclopia is a lethal autosomal recessive trait. Gorlin et al. ${ }^{18}$ suggested that some cases of holoprosencephaly may be caused by an autosomal recessive gene in an individual with normal karyotype, and suggested that the responsible gene is located on the short arm of chromosome 18.

In our case chromosomal studies were normal. Karseras and Laurence ${ }^{19}$ concluded that normal chromosomal studies do not necessarily exclude an underlying chromosomal disorder, since this may not have been discovered by earlier methods and may still escape detection by current methods.

Jaschevatzky et al. ${ }^{5}$ discussed the presence of extracephalic and extrafacial malformations in cyclopia with trisomy-D. They reported detailed information about these malformations in each case in the literature known until 1976 with chromosomal aberrations. In cases with normal karyotype there was no information about extracephalic malformations. In our case the only extracephalic defect was persistent patent ductus arteriosus.

A classification of two main aetiological groups was suggested by Taysi and Tinaztepe. ${ }^{21}$ In the first group there were only cephalic malformations and the karyotype was normal. In the second group there were cephalic as well as extracephalic malformations with anomalous karyotype, mostly trisomy-D. Our case with patent ductus arteriosus and normal karyotype does not seem to belong to any of the aforementioned groups, so the discussion is left open at this point.

The environmental factors known to cause cyclopia in clinical practice and in experimental investigations are numerous. Mollica et al. ${ }^{3}$ reported six cases of cyclopia in which the mother was exposed to drugs or to other potentially teratogenic environmental factors during organogenesis. These factors were ionising radiation, contraceptives, viraemia plus cortisone and salicylate, rubella vaccine, antibiotics, and amidopyrine. Other known factors are lithium, magnesium, veratrum alkaloids (in ruminantes) vinca alkaloids, and vitamin $A$ in rats. ${ }^{1}$

In our case the pregnancy developed under an intrauterine device containing copper, which was inserted one week after the last menstrual period; the mother was also given drugs during organogenesis (tranexamic acid and ampicillin). There is no mention in the literature of cases of cyclopia after 
tranexamic acid ingestion, but antibiotics were incriminated by Mollica et al. ${ }^{3}$ We found no reports of intrauterine devices as the possible cause of cyclopia.

On histopathological examination of the eyeball the main finding was the presence of a well formed globe in which malformations are prominent: hypercellular cornea, non-cleavage anterior chamber angle, lack of dilator muscle and endothelial cells of the iris, anterior insertion of the ciliary body, anomalous transformation of ciliary pigment epithelium into retinal pigment epithelium, choroidal colobomas, disorganisation and dysplasia of the retina with rosette formation, lack of retinal ganglion cells and nerve fibres, and projection of retinal tissue into the vitreous and into the sclera.

Most of these malformations were discussed or mentioned by other authors ${ }^{1214}$ in cases of cyclopiasynophthalmos. The anterior insertion of the ciliary body processes (Figs. 4 and 5) was discussed by Barishak $^{21}$ in the human embryo and in avian and mammalian eyes but has not previously been reported in cyclopian eyes. Barishak concluded that anterior insertion of the ciliary processes is normally found in the human embryo during the second trimester of pregnancy. In the third trimester the ciliary processes move backward posterior to the apex of the angle of the anterior chamber, and this is the result of a difference in growth rate of the neuroepithelial and mesodermal layers at the ciliary body region.

\section{References}

1 Torczynski E, Jacobeic FA, Johnston MC, Font RL, Madewell JR. Synophthalmia and cyclopia: a histologic, radiographic and organogenetic analysis. Doc Ophthalmol 1977;44:311-78.

2 Duke-Elder S. Anomalies of fusion. System of ophthalmology. St Louis: Mosby, 1963; 3: 429-49.
3 Mollica F, Pavone L, Sorge G. Maternal drug ingestion and cyclopia. J Pediatr 1981; 98: 680.

4 Benawra R, Mangurten HH, Duttell DR. Cyclopia and other anomalies following maternal ingestion of salicylates. $J$ Pediatr 1980; 96: 1069-71.

5 Jaschevatzky OE, Goldman B, Georghion P, Grunsterin S, Pevzner S. Trisomy-D in a cyclops with cardiovascular defects. Acta Obstet Gynecol Scand 1976; 55: 73-6.

6 Arakaki DT, Waxman SH. Trisomy-D in cyclops. J Pediatr 1969; 74: 620-5.

7 Batts JA, Punnet HH, Valdes-Dapena M, Coles JW, Green WR. A case of cyclops. Am J Obstet Gynecol 1972; 112: 65761.

8 Cohen MM. Chromosomal mosaicism associated with a case of cyclopia. Pediatrics 1966; 69: 793-8.

9 Halbrecht I, Kletzky O, Komlos L, Lotker M, Gersht N. Trisomy-D in cyclops, a case report. Obstet Gynecol 1971; 37: 391-3.

10 Khudr G, Olding L. Cyclopia. Am J Dis Child 1973; 125: 120-2.

11 Cohen MM, Gorlin RJ. Genetic consideration in a sibship of cyclopia and clefts. Birth Defects $1969 ; 5$ : 113-8.

12 Zimmer EZ, Divon MY, Halperin M, Peretz BA. Cyclopia with trisomy-D. Eur J Obstet Gynecol 1982; 13: 215-9.

13 Towes HA, Jones HW Jr. Cyclopia in association with trisomy-D and gonadal agenesis. Am J Obstet Gynecol 1968; 102: 53-6.

14 Barber AL, Muelling RJ Jr. Cyclopia with complete separation of the neural and mesodermal elements of the eye. Arch Ophthalmol 1950; 43: 989-1003.

15 Adelman HB. The problem of cyclopia. $Q$ Rev Biol 1936; 11: 161-82, 248-304.

16 De Myer WE, Zeman W, Palmer CG. The face predicts the brain: diagnostic significance of median facial anomalies for holoprosencephaly. Pediatrics 1964; 34: 256-263.

17 Francois J. Heredity in ophthalmology. St Louis: Mosby, 1961: 173-6.

18 Gorlin RJ, Yunis J, Anderson VE. Short arm deletion of chromosome 18 in cebocephaly. Am J Dis Child 1968; 115: 473-6.

19 Karceras AG, Laurence KM. Eyes in arinencephalic syndromes. Br J Ophthalmol 1975; 59: 462-73.

20 Taysi K, Tinaztepe K. Trisomy-D and the cyclops malformation. Am J Dis Child 1972; 124: 710-3.

21 Barishak YR. Development of the angle of the anterior chamber of the eye in animals and human embryos. Metab Ophthalmol 1978; 2: 269-73. 